International Journal of Robotic Computing

Vol. 1, No. 1 (2019) 55-79

(C) KS Press, Institute for Semantic Computing Foundation

DOI: $10.35708 / \mathrm{RC} 1868-126251$

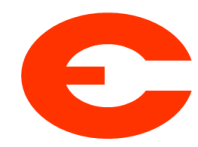

\title{
Automotive Radar-based Self Localization Using Navigation Maps for Autonomous Driving
}

\author{
Ahmad Pishehvari ${ }^{1,2}$, Uri Iurgel ${ }^{2}$, Stephanie Lessmann² ${ }^{2}$ Lutz Roese-Koerner ${ }^{2}$, \\ and Bernd Tibken ${ }^{1}$ \\ 1 Automation/Control technology, Bergische Universität Wuppertal, Gausstraße 20 \\ Wuppertal, North Rhein-Westphalia, Germany \\ ahmad.pishehvari@yahoo.com \\ 2 Aptiv Services Deutschland GmbH, Am Technologiepark 1 Wuppertal, North \\ Rhein-Westphalia, Germany \\ \{uri.iurgel, stephanie.lessmann, lutz.roese-koerner\}@aptiv.com
}

Received (11/02/2018)

Revised (06/18/2019)

Accepted (07/15/2019)

\begin{abstract}
This paper describes a framework for precise self-localization using 2D radar scan matching based on a digitalized map. For this purpose, radars, odometers, a gyroscope and a global digital map are combined. Basically estimated ego-motion using motion sensors is improved using a novel scan matching approach in order to attain globally corrected self-localization results. The matching process is based on map information, iterative optimization using the Gauß-Helmert-Model and two novel weighting methods to register the environment map using radar information. This approach focuses on self-localization in a global frame without using Global Navigation Satellite Systems (GNSS).

Beside our main innovation of using native non-discretized map lines for matching we also apply two novel weighting methods to cope with noisy radar scans for matching problem. By applying the Gauß-Helmert-Model we also consider the individual measurement uncertainties to make the approach even more robust against noisy data. Using map lines and data points categorizes our approach in the point-to-feature scan matching family. The performance and usability of the proposed approach is evaluated in real-world experiments and compared qualitatively and quantitatively to the state of the art matching methods.

The results illustrate an improvement in precision and computational demand compared to other point cloud based matching methods.
\end{abstract}


Keywords: Radar based self-localization; Scan matching; Navigation maps.

\section{Introduction}

Accurate ego-motion estimation is a fundamental challenge and one of the most important requirements for mobile robot applications and autonomous driving systems. For obstacle detection and avoidance as well as for mapping and motion tracking systems a mobile robot or a vehicle must retain its own pose (position and azimuth). The ego-motion is basically retrieved in this approach by mechanical motion sensors using a gyro and four wheel-based odometers (dead reckoning), included as standard features in our test vehicle. However, the calculated ego-trajectory using these sensors is not accurate enough for autonomous vehicle applications.

Inertial sensors are highly affected by drift and wheel-based encoders are not accurate enough due to wheel slippage and imperfection of motion models. This leads to a large error, which accumulates over time. One of the strategies to mitigate this inaccuracy is scan matching, which is used in this approach to improve the ego-motion estimated basically by dead reckoning. In scan matching the pose of a sensor observation is investigated by seeking its best overlap with a reference. If this reference is another sensor scan assuming a known initial pose, a local scan matching is performed [18] and it can be an alternative to dead reckoning for retrieving the ego-motion. However, in our approach the estimated ego-motion by dead reckoning is improved by searching for an alignment between radar measurements and a digital map. Since we assume a roughly known initial pose it results also in a local scan matching in a global coordinate frame [27]. The first step and the most computationally expensive part of matching is to find the correspondences between data and model. Compared to other matching methods it is considerably less complex in calculation in this approach, since our model (digital map) consists of lines and for each radar detection its nearest map line is its correspondent feature. Afterwards a filtering method is applied to reduce the effect of objects, which do not reflect static or realistic landmarks according to the map information (e.g. objects behind nontransparent map objects). Finally, Euclidean distance between data and their correspondent model features are minimized iteratively by applying the Gauß-Helmert-model (GHM), which considers the known data covariances and makes the solution robust against noisy data.

Additionally, two novel weighting criteria can be used to weight detections higher, which are more probable to be non-noisy data. It makes the approach even more robust against uncertainties. Both weighting methods can be applied separately or in combination and integrated in the GHM. Fig. 1 shows the result of a test drive in a parking lot with and without applying scan matching. The ego-trajectory (blue) using dead reckoning in Fig. 1.a is biased, which leads to generation of an inconsistent occupancy grid map (OGM). After applying the proposed scan matching a more accurate result is achieved in Fig. 1.b with a 


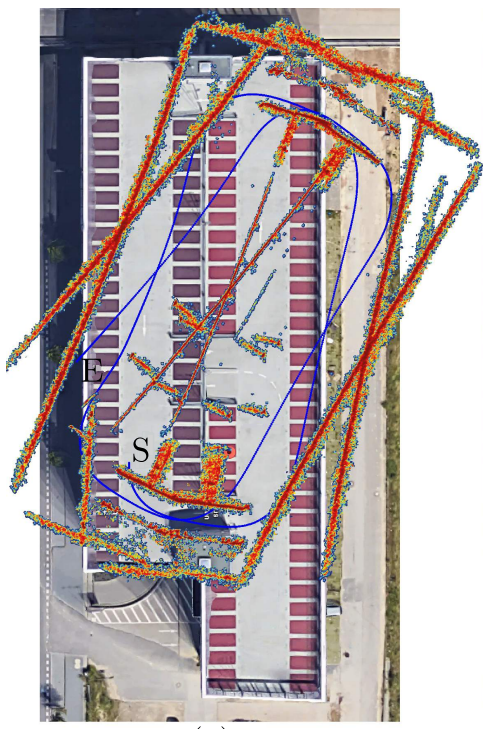

(a)

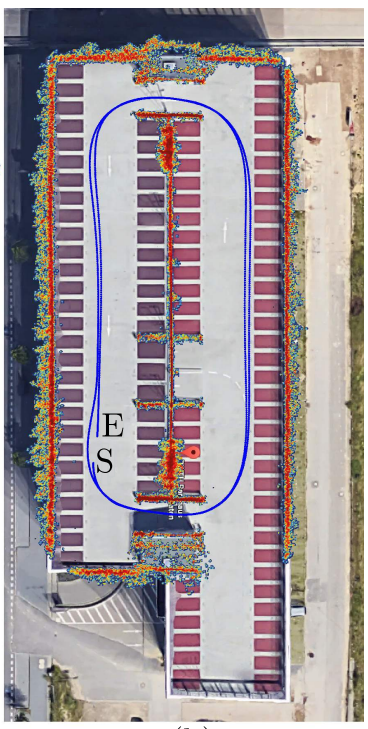

(b)

Fig. 1: The ego-pose estimation of a test vehicle by dead reckoning (a) and the improved ego-motion estimation by scan matching (b) in blue. Based on these trajectories an occupancy grid map is generated. The letters $\mathrm{S}$ and $\mathrm{E}$ show the start and end point, respectively. Photo: Imagery (C)2018 Google, Map data (c)2018 GeoBasis-DE/BKG (@2009).

consistent OGM, which overlaps completely with the environment representation. For the proposed method in this paper two European patent applications with the numbers EP 18153440.5 and EP 18153439.7 are already filed. This is an extended version of the article Radar Scan Matching Using Navigation Maps published in proceedings of IRC 2019 [23]. This paper is organized as follows. Section 2 reviews related works. Section 3 presents digital map data processing. Section 4 describes the scan matching algorithm in detail. Section 5 demonstrates experimental results, which are evaluated qualitatively and quantitatively to validate our approach. Finally, Section 6 concludes this paper and discusses future work.

\section{Related work}

Retrieving the ego-motion is widely proposed using different sensors such as GNSS. Some of these systems can achieve an acceptable accuracy level (within centimeters) in open sky. However, due to the limited access to satellite signals GNSS networks are not usable in some areas such as tunnels or undergrounds or where very strong multi-path propagation occurs e.g. areas surrounded by tall glass covered buildings. Dead reckoning is one of the alternatives in such areas, its operation is prone to be affected by systematic and non-systematic errors. In [4] some methods are introduced to cope with systematic errors. However, 
mechanical motion sensors are often affected by non-systematic errors such as noise causing a significant degradation of self-localization quality.

Using vision sensors [15] or remote sensing systems such as LiDAR (light detection and ranging) [9] and radar (radio detection and ranging) various techniques can also be applied for tracking the ego-pose. Scan matching is one of the widely used algorithms, which can be employed in different ways to retrieve the vehicle's ego-state or to improve it by estimating only its displacement. A possible approach for ego-pose retrieving is to match two successive sensor observations. In [20] visual odometry is used to reconstruct the ego-pose by analyzing the changes that motion induces on the consecutive image features. However, the weather- and light-conditioned performance of vision-based systems limits their usage for environment sensing.

Using remote sensors, the matching methods can also be applied for the registration problem of two consecutive observations, which can be categorized in optimization-based and probabilistic methods. Correlative scan matching is an example of probabilistic algorithms, which maximizes the probability of having observed the data given the last one and is applied in [22] for laser scan matching. Iterative closest point (ICP) [2], Hough transformation [7], FourierMellin transform [8], histograms [5] and normal distribution transform (NDT) [3] are possible algorithms for optimization-based matching. ICP algorithm and its variants such as point-to-line ICP (PLICP) [6], MbICP (metric based ICP) and IDC (iterative dual correspondences) belong to the most pervasively applied techniques for scan matching. MbICP and IDC are used in [19] and [18], respectively, for retrieving the ego-pose using laser scanner. The motivation of using radars is -in contrast to LiDAR - their resilience to adverse weather conditions in outdoor areas. However, radars tend to be more affected by noise and multiple reflections and because of their sparse measurements the matching of consecutive scans is less researched. Instead, radar observations can be compared with a predefined map to improve the ego-pose estimated basically by other sensors e.g. dead reckoning. The environment map can be a probabilistic map generated based on sensor data (vision, laser or their combination) [17] or it can be a navigation map such as Google map or OpenStreetMap (OSM) [12]. Using sensor-based environment model results in big volumes of data to be stored and processed and demands much more time and effort than using a navigation map. Environment models are employed widely in literature for localization problem e.g. OSM is used in [25] in combination with LiDAR data for self-localization. SLAM (Simultaneous localization and mapping) is another approach for retrieving the ego-position while constructing a map of the environment [26] without using a priori map. It requires landmark extraction for loop closure which makes the SLAM computationally more complicated in comparison with scan matching. In addition, the feature extraction is inaccurate in the case of using radars. There are also SLAM variants with a fast performance but still the simplicity and low computational cost of the proposed scan matching is a major advantage.

Motivated by the mentioned works, we propose a simple real-time matching 
technique with low computation time using an efficient environment map and compare it with some of the state of the art algorithms. The next section describes how the navigation map can be used for matching problem.

\section{Map representation}

In order to refine the ego-position, which is basically estimated by dead reckoning the required environmental information is obtained from an a priori map. It can be any kind of $2 \mathrm{D} / 3 \mathrm{D}$ map in a local or a global coordinate frame with desired geometric representation complexity. However, the use of $2 \mathrm{D}$ environment maps for matching is computationally more efficient. The free OSM is one of the possibilities for using navigation maps. A segment of such map is shown in Fig. 2. To attain a global ego-pose tracking OSM is used in this approach. For mapmatching solely the landmarks such as buildings, roadsides, etc. are required. These are shown in Fig. 2 as purple lines, which are described mathematically as native discrete line segments for the matching problem.

\section{Scan matching process}

Scan matching is the process of calculating a rigid body transformation of a sensor measurement to maximize its overlap with a reference e.g. a navigation map. This conversion consists of a longitudinal $\left(t_{x}\right)$, lateral $\left(t_{y}\right)$ and rotational transformation $(\phi)$, which can be represented by a displacement vector $\underline{\mathrm{d}}=\left(t_{x}, t_{y}, \phi\right)^{T} \in \mathbb{R}^{3 \times 1}$. The first and most computationally intensive step of a matching algorithm is to find correspondences between data and model. The displacement of radar measurements relative to the map cannot be large, since the time between receiving two successive scans is in millisecond range and the matching process is performed continuously. Based on this assumption the correspondent map line segment for each detection is expected to be its nearest line in the map. In this manner the scan matcher does not need to perform the

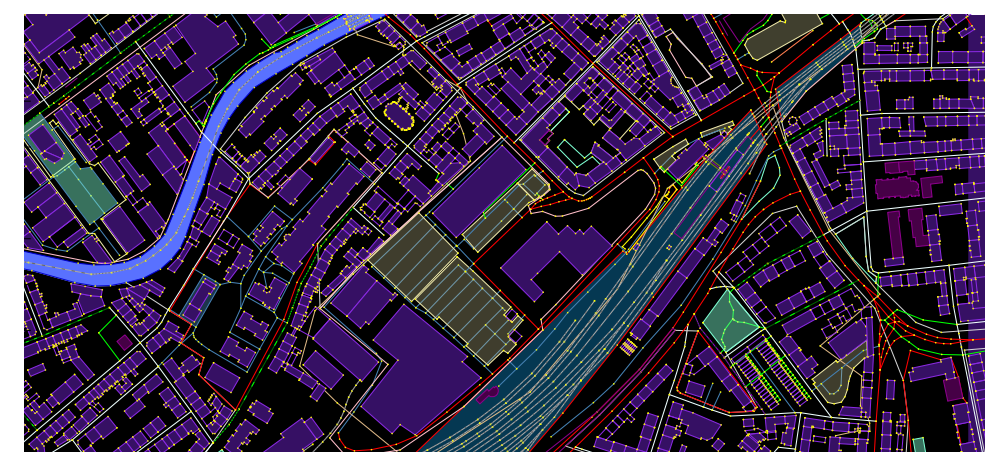

Fig. 2: An example for environment representation. (Data from OpenStreetMap). 
computationally expensive feature extraction, which can be also inaccurate according to the high noise level of radar measurements. To make the approach robust against noisy data the radar detections are filtered according to the distance from their correspondent map lines. A weighting criterion is then used to reduce the influence of false detections, which makes the approach even more robust against noisy radar data.

Fig. 3 illustrates the iterative algorithm for ego-pose estimation using radar scan matching containing the entire process. The matcher iterates until the modification of displacement vector $(\Delta \underline{d})$ is less than a threshold $\underline{\epsilon}$. The position $(x, y)$ and orientation $(\theta)$ of the vehicle are described by a state triple $\underline{p}_{\text {vehicle }}=(x, y, \theta)^{T}$. A state increment between radar scan and map is calculated by the matcher, which reduces the scan displacement. The result of matching is a rigid body transformation, which corrects the inaccurate ego-pose estimated by dead reckoning.

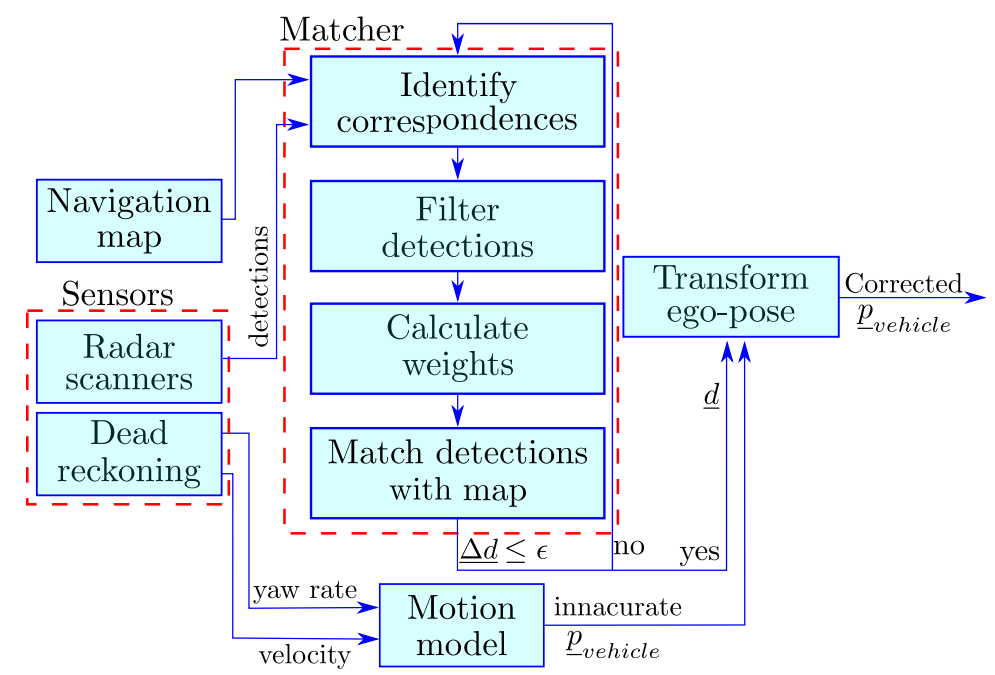

Fig. 3: Algorithm of Ego-pose estimation applying proposed scan matching.

In Fig. 4 the blue points are the detections of a single radar scan rendered from ego-pose estimated by dead reckoning. The position and the orientation of the ego vehicle are shown as a black rectangle and a red arrow, respectively. In Fig. 4.b a slight displacement of this scan relative to the map can be identified. Scan matcher seeks the best possible transformation that minimizes iteratively the displacement. It improves the ego-motion estimation, which is illustrated in Fig. 4.c.

In the following section the filtering and weighting of the radar detections are described. 


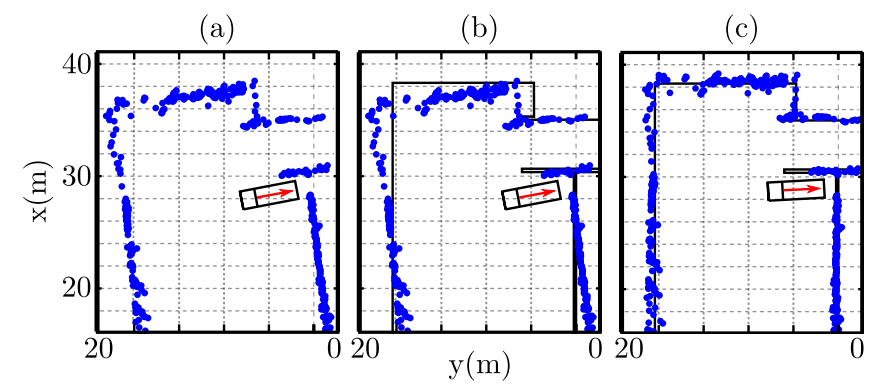

Fig. 4: Ego-pose improvement using scan matching. The radar detections (blue) rendered from ego-pose estimated by dead reckoning (a) can be compared with the map (b). The ego-pose is corrected applying scan matching (c).

\subsection{Filtering of radar detections}

Optimization-based matching is sensitive to noise, occlusion and spurious data (outliers), such as multiple reflections or false detections. An essential part of scan matching is to reject this noisy data to reduce their effect on the result. By a priori map information we can filter non-static objects which are far away from available landmarks or those behind nontransparent map objects. In this section two filtering methods are introduced which use the environment map and the data association between map and sensor observations as input.

\section{Thresholding-based filtering}

Applying hard thresholding the perpendicular distance of the observation form their corresponding map line is considered. If this distance $d_{\perp}$ exceeds a threshold $S_{t h}$ the observation is filtered. To avoid the need for a highly accurate initial pose estimation this threshold has to be selected initially wider and it can be reduced over time after initialization. The large displacement during initialization is allowed because of the iterative solution of the GHM. The correspondences have to be re-identified after each iteration. Due to the continuously matching in millisecond range the ego-trajectory cannot have a large drift. Based on this assumption the ego-pose has always a small displacement and the radar measurements can be filtered in this way.

For scan matching we assume a known initial pose which can be estimated by global localization algorithms such as particle filtering or by using GPS, etc. However, if the initial pose estimation deviates significantly from the ground truth, a hard thresholding could result in filtering of non-noisy data. This filtering method demands very low computation time but if the initial pose error exceeds certain limits the scan matcher is not able to converge to the correct pose. In such a case there is no chance to recover the correct pose because the assumption is a small pose estimation error due to the continuous map matching. 
Another worst-case scenario happens if during a long period of time there are no observations to be matched with the map. This can occur if the observation contains static detections which are not corresponding to the map e.g parked vehicles. Missing objects in the map lead also to a failed correspondences identification. In this case the accumulation of pose estimation error caused by dead reckoning can result in a large distance between observations and their corresponding map features (lines). Applying a hard thresholding could lead also in this case to the filtering of useful sensor information and in worst case to losing the ego-pose in the map frame. It is therefore recommended to use robust estimators for filtering which is described in the following.

\section{Robust estimation}

Using the thresholding-based filtering is due to its computational simplicity advantageous. However, this method is critical regarding to its robustness against the inaccurate pose estimation. Since OSM consists solely of lines, a line fitting algorithm can be used for filtering such as least-squares estimator, RANSAC $^{3}[10]$ or MSAC $^{2}$ [28]. In each sample time a 2D set of sensor measurements $\underline{P}=\left(\begin{array}{llll}\underline{p}_{1} & \underline{p}_{2} & \cdots & \underline{p}_{m}\end{array}\right)^{T} \in \mathbb{R}^{m \times 2}$ and a map consisting of $n$ lines $\mathcal{L}_{r}, r=1,2, \ldots, n$ are available. For filtering based on line fitting each time only the sensor observations which are correspondent with a single map line $\mathcal{L}_{r}$ are considered, so that $n$ models have to be estimated for the entire sensor observation. The problem of line fitting is to find a line which minimizes the sum of perpendicular distances which are referred to as orthogonal regressions. Least-squares estimator is one option to solve this problem. However, if the nonmodeled noise has a high proportion this estimator does not perform robustly since this algorithm is not able to classify the noisy data. Robust estimators use only a portion of data which are called inlier and entail the close points to the model response. To classify the data as inlier and outlier it is assumed that the information about process noise scale is available.

The idea of RANSAC is to select two random samples and define a line equation

$$
\underline{n}_{p_{j}}-b=0, p_{j} \in \mathcal{P}
$$

where $\underline{n}$ is the unit normal vector of the corresponding map line and $b$ is the perpendicular distance of the line from the origin. Based on this equation the model

$$
M=\left[n_{x} n_{y} b\right]
$$

can be defined. The set of sensor measurement which corresponds with the line $\mathcal{L}_{r}$ is described as $\mathcal{P}$ with

$$
\mathcal{P}=\left\{p_{j} \mapsto \mathcal{L}_{r}, \quad 1 \leq j \leq m\right\}
$$

\footnotetext{
${ }^{3}$ RANdom SAmple Consensus

${ }^{2}$ M-estimator SAmple and Consensus
} 


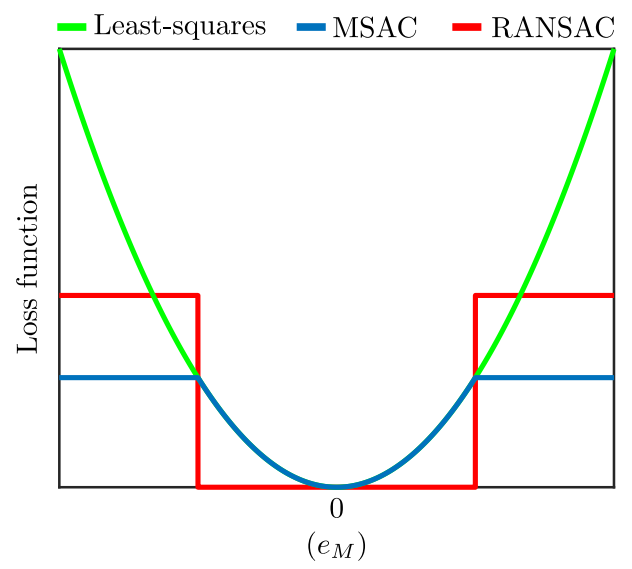

Fig. 5: Comparison of loss function for different model fitting algorithms.

RANSAC selects two points randomly from the samples in $\mathcal{P}$ and estimates a model $M_{r}, n=1, \ldots, n$. Then the samples have to be classified to inliers and outliers based on a pre-defined threshold $C$ base on process noise. The model with the highest number of inliers is finally selected as the most probable hypothesis. Then it solves the model fitting problem as an optimization problem defined by

$$
\hat{M}=\underset{M}{\operatorname{argmin}}\left\{\sum_{\mathcal{P} \in \underline{P}} e_{M}(\mathcal{P}, \mathcal{M})\right\}
$$

where $\mathcal{M}$ is the model (map). The parameter $e_{M}$ defines the error which is the perpendicular distance between an observation and the estimated line by line fitting, i.e.

$$
e_{M}=\underline{n} \underline{p}-b .
$$

The loss function of RANSAC is defined the by

$$
\operatorname{Loss}(\mathcal{P}, M)= \begin{cases}0 & \left|e_{m}\right|<\sigma \\ C & \text { otherwise }\end{cases}
$$

associated with the measurement $\mathcal{P}$ regarding to the defined model. As it can be considered in the loss function all inliers are handled equally independent form their error $e_{M}$. A robust M-estimator with the loss function

$$
\operatorname{Loss}(\mathcal{P}, M)= \begin{cases}e_{m}^{2} & \left|e_{m}\right|<C \\ C^{2} & \text { otherwise }\end{cases}
$$

includes also all inliers but weights them according to their error regarding to the estimated model in contrast to RANSAC. Figure 5 shows the difference of loss functions for the discussed model fitting approaches and their behavior to cope with outliers. The following algorithm is used in this work for filtering by robust estimation methods. As termination criterion a maximum number 

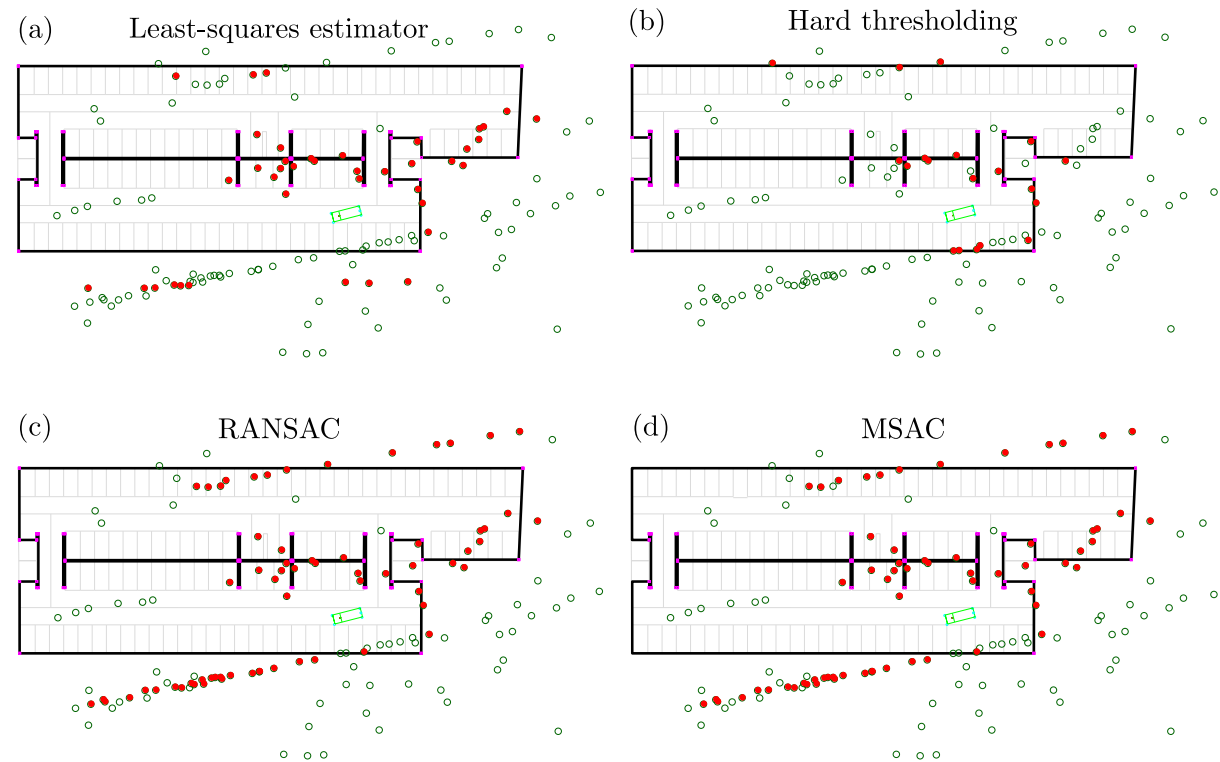

Fig. 6: Four different methods used for filtering of one sensor observation. Green singles are the unfiltered data and red dots shows the result of filtering. Black solid lines represent the OSM lines.

of iterations can be used. In Fig. 6 the results of applying hard thresholding and robust estimation for a single radar observation are illustrated. As it can be observed in this figure if the pose deviates considerably from its true value then the overlap between the map and the observation reduces strongly. As shown in Fig. 6.b the hard-thresholding method filters a considerable amount of non-noisy information. The robust estimators in 6.c and 6.d have significantly better performance since they use the inlier/outlier classification. In this case both robust estimators output the same result. In contrast, the least-squares estimator in Fig. 6 is using the entire data making it not robust enough to cope with highly noisy data.

\subsection{Scan matcher}

The main objective of the scan matcher is to use correspondences between radar detections and map line segments to find a transformation. It minimizes the total 2D squared distances between detections and their correspondent lines. The distance between a data point $\underline{p}_{i}, i=1, \ldots, m$ and a map line $\mathcal{L}_{j}, j=1, \ldots, n$ can be described as

$$
\delta_{i}=\underline{n}_{j} \cdot \underline{p}_{i}-b_{j}
$$

where $\underline{n}_{j}$ is the unit normal vector of the corresponding map line and $b_{j}$ is the perpendicular distance of the line from the origin. A $2 \mathrm{D}$ rotation matrix $R$ and 
$2 \mathrm{D}$ translation vector $t$ expressed by

$$
\underline{R}(\phi)=\left(\begin{array}{cc}
\cos (\phi) & -\sin (\phi) \\
\sin (\phi) & \cos (\phi)
\end{array}\right), \underline{t}=\left(\begin{array}{l}
t_{x} \\
t_{y}
\end{array}\right)
$$

shall be estimated to minimize the mentioned distances $\delta_{i}$. The functional relationship

$$
F(\underline{P}, \underline{d})=\sum_{i=1}^{m}\left[\underline{n}_{i}^{T}\left(\underline{R} \underline{p}_{i}+\underline{t}\right)-b_{i}\right]^{2}
$$

has to be minimized to find the optimum solution for the matching problem, which contains the total squared Euclidean distances of data points and map lines. Considering the assumption of small displacement of radar scans with regard to environment model the nonlinear rotation matrix can be approximated by the first order term in $\phi$ resulting

$$
\sin (\phi)=\phi, \quad \cos (\phi)=1 \Rightarrow \underline{R}(\phi)=\left(\begin{array}{cc}
1 & -\phi \\
\phi & 1
\end{array}\right) .
$$

A Cartesian coordinate system is used for the map and radar measurement representation. As the range and azimuth of radar detections contain known, range dependent uncertainties we aim to solve a weighted least squares problem to obtain $\underline{d}$. The used radars are more precise in the range measurement $r$ than in the azimuth $\phi$ resulting in a long narrow ellipse in polar coordinates, transverse to the target line of sight. Taking the uncertainties into account the matching problem can be solved using the Gauss-Markov-model if there are no multiplicative dependencies between observation components and parameters to be estimated. With expanding the functional relationship in 10 to

$$
\begin{aligned}
& F(\underline{P}, \underline{d})=\sum_{i=1}^{m}\left[n_{x i} P_{x i}+\phi n_{y i} P_{x i}-\phi n_{x i} P_{y i}+n_{y i} P_{y i}+t_{x} n_{x i}+t_{y} n_{y i}-b_{i}\right]^{2} \\
& \left.=\sum_{i=1}^{m}\left[\begin{array}{lll}
n_{y i} P_{x i}-n_{x i} P_{y i} & n_{x i} & n_{y i}
\end{array}\right)\left(\begin{array}{c}
\phi \\
t_{x} \\
t_{y}
\end{array}\right)+n_{x i} P_{x i}+n_{y i} P_{y i}-b_{i}\right]
\end{aligned}
$$

it can be however considered, that there exist a multiplicative dependency between some of the observation components and the parameters to be estimated. In such a case we have two possibilities to solve the optimization problem namely Total Least Squares introduced by [11] and Gauss-Helmert-model. Due to the need for singularity decomposition in total least squares this is a more time consuming approach. Compared to this GHM is more complicated formulated but its application leads to a faster solution calculation. We will use a GHM [14, p. 212] in order to completely utilize all known uncertainties. 


\section{Gauss-Helmert-Model}

In this section the unconstrained formulation of the Gauss-Helmert-model is explained. As the mathematical context of the functional relationship in 10 is nonlinear, the model construction has to be linearized first using a Taylor approximation. Subsequently, the parameters of the nonlinear approach can be calculated iteratively. Fig. 7.a illustrates the covariance ellipses of three detections and their projection areas in the map, which are considered in the GHM. Given is an observation vector

$$
\underline{P}=\left(\underline{p}_{1} \underline{p}_{2} \cdots \underline{p}_{m}\right)^{T} \in \mathbb{R}^{m \times 2}, \underline{p}_{i}=\left(p_{i x} p_{i y}\right)^{T} \in \mathbb{R}^{2 \times 1}
$$

containing $m$ radar detections. The observations $\underline{P}$ are assumed to be normally distributed with known covariance matrices

$$
\underline{\Sigma} \in \mathbb{R}^{2 m \times 2 m}
$$

i.e.

$$
\underline{\mathcal{P}} \sim N(\underline{A} \underline{\xi}, \underline{\Sigma}) \text { with } \underline{\xi} \text { as true parameter vecor. }
$$

The variance covariance matrix $\underline{\Sigma}$ is block-diagonal with

$$
\underline{\Sigma}_{i}=\left(\begin{array}{cc}
\sigma_{i x x} & \sigma_{i x y} \\
\sigma_{i y x} & \sigma_{i y y}
\end{array}\right), i=1, \ldots, m
$$

as diagonal elements. The assumption is that there are $b$ non-linear relationships between one or more observation(s) and up to 3 unknown parameters

$$
F(\underline{P}, \underline{d}) \stackrel{!}{=} 0
$$

which is used as equality constraint for the optimization problem [24]. For the linearization of the functional relationship in (10), the optimal solution $\underline{d}^{*}=$ $\left(t_{x}^{*}, t_{y}^{*}, \phi^{*}\right)^{T}$ and the measurement $\underline{P}$ has to be split up into

$$
\begin{aligned}
& \underline{\tilde{P}}=\underline{P}+\underline{\mathcal{V}}=\underbrace{\underline{P}-\underline{P}_{0}}_{\underline{\underline{P}}}+\underline{P}_{0}+\underline{\mathcal{V}}=\underline{P}_{0}+\underline{\Delta P}+\underline{\mathcal{V}}, \\
& \tilde{d}^{*}=\underline{d}_{0}^{*}+\underline{\Delta d^{*}} .
\end{aligned}
$$

A Taylor point

$$
\underline{z}_{0}=\left[\underline{d}_{0}^{*}, \underline{P}_{0}\right]^{T}
$$

related to both searched parameters and sensor observations must be chosen [16]. The random variable $\underline{\mathcal{V}}$ is an improvement term. Linearization using a Taylor 
series and the Taylor point in 20 results in

$$
\begin{aligned}
& F\left(\underline{\tilde{P}}, \underline{\tilde{d}}^{*}\right)=F\left(\underline{P}_{0}+\underline{\Delta P}+\underline{\mathcal{V}}, \underline{d}_{0}^{*}+\underline{\Delta d^{*}}\right) \\
& =\underbrace{\left.F\left(\underline{P}, \underline{d}^{*}\right)\right|_{\frac{P=P_{0}}{\underline{d^{*}}=\underline{d}_{0}^{*}}}}_{\underline{w}_{0}}+\underbrace{\left.\nabla_{P} F\left(\underline{P}, \underline{d}^{*}\right)\right|_{\frac{P}{\underline{P}=\underline{P}_{0}}} ^{\underline{\underline{d}^{*}=\underline{d}_{0}^{*}}}}_{\underline{B}^{T}}
\end{aligned}
$$

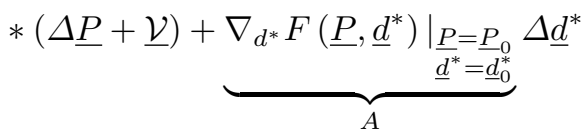

$$
\begin{aligned}
& =\underline{w}_{0}+\underline{B}^{T}(\Delta \underline{P}+\underline{\mathcal{V}})+\underline{A} \Delta \underline{d}^{*} \\
& =\underbrace{\underline{w}_{0}+\underline{B}^{T} \underline{\Delta P}}_{\underline{w}}+\underline{B}^{T} \underline{\mathcal{V}}+\underline{A} \underline{\Delta d^{*}} \\
& =\underline{w}+\underline{B}^{T} \underline{\mathcal{V}}+\underline{A} \underline{\Delta d^{*}}=0 \text {. }
\end{aligned}
$$

This equality is the constraint of the cost function

$$
\operatorname{Min}_{\underline{\mathcal{V}}} \underline{\mathcal{V}}^{T} \Sigma^{-1} \underline{\mathcal{V}}
$$

which means that the the improvement term $\underline{\mathcal{V}}$ has to be minimized. The elements of

$$
\underline{w}_{0}=\left(w_{01}, w_{02}, \ldots, w_{0 m}\right)^{T}
$$

in this equation result from (10) evaluated with $\underline{p}_{i 0}$ and $\underline{d}_{0}^{*}=(0,0,0)^{T}$. Each row of $\underline{A} \in \mathbb{R}^{m \times 3}$ and each element of matrix $\underline{B} \in \mathbb{R}^{m \times 2 m}$ contains the gradient vector of (10) with respect to $\underline{d}$ and $\underline{P}$, respectively, evaluated with $\underline{p}_{i 0}$ and $\underline{d}_{0}^{*}$. Finally, the weighted least-squares adjustment

$$
\begin{array}{ll}
\text { Objective function: } & \Phi(\underline{\mathcal{V}})=\operatorname{Min}_{\underline{\mathcal{V}}} \underline{\mathcal{V}}^{T} \Sigma^{-1} \underline{\mathcal{V}} \\
\text { Contraints: } & \underline{B^{T}} \underline{\mathcal{V}}+\underline{A} \underline{\Delta} d^{*}+\underline{w} \\
\text { optimization variable: } & \underline{\mathcal{V}} \in \mathbb{R}^{m \times 2}, \underline{\Delta d^{*}} \in \mathbb{R}^{3}
\end{array}
$$

can be formulated as Gauss-Helmert-Model. The Lagrangian is used for computing of necessary and sufficient optimality conditions of this optimization problem. This is described by

$$
L\left(\underline{\mathcal{V}}, \underline{\Delta d^{*}}, \underline{k}\right)=\underline{\mathcal{V}}^{T} \Sigma^{-1} \underline{\mathcal{V}}-2 \underline{k}^{T}\left(\underline{B}^{T} \underline{\mathcal{V}}+\underline{A} \underline{\Delta d^{*}}+\underline{w}\right)
$$


whereby $\underline{k}$ contains $p$ Lagrange multipliers. Setting the gradients to zero

$$
\begin{aligned}
\nabla_{\underline{\mathcal{V}}} L\left(\underline{\mathcal{V}}, \underline{\Delta d^{*}}, \underline{k}\right) & =2 \Sigma^{-1} \underline{\mathcal{V}}-2 \underline{B} \underline{k}^{T} \stackrel{!}{=} 0 \\
\nabla_{\underline{\Delta d^{*}}} L\left(\underline{\mathcal{V}}, \underline{\Delta d^{*}}, \underline{k}\right) & =-2 \underline{A}^{T} \underline{k} \stackrel{!}{=} 0 \\
\nabla_{\underline{k}} L\left(\underline{\mathcal{V}}, \underline{\Delta d^{*}}, \underline{k}\right) & =-2\left(\underline{B}^{T} \underline{\mathcal{V}}+\underline{A} \underline{\Delta d^{*}}+\underline{w}\right) \stackrel{!}{=} 0
\end{aligned}
$$

results in the first oder optimality conditions. These conditions lead to

$$
\begin{aligned}
\underline{\mathcal{V}} & =\Sigma \underline{B} \underline{k} \\
\underline{A}^{T} \underline{k} & =0 \\
\underline{B}^{T} \underline{\mathcal{V}}+\underline{A} \underline{\Delta d}^{*}+\underline{w} & =0 .
\end{aligned}
$$

By inserting (31) in (33) the equation

$$
\underline{B}^{T} \Sigma \underline{B} \underline{k}+\underline{A} \underline{\Delta d^{*}}+\underline{w}=0
$$

is obtained. On the basis of normal equations in (34) and 32 a linear system

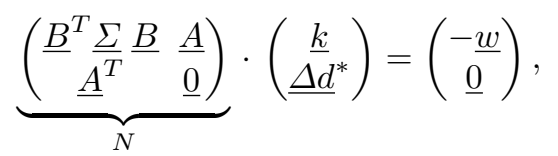

can be formulated. The solution of this linear system results in a estimation for $\Delta d^{*}$. Due to the linearization of this system, iterations according to the Gauss-Newton method may be needed to estimate $\underline{d}^{*}[21]$. In each iteration the data association for finding correspondences, filtering and weighting have to be performed. As termination criterion a lower bound for the improvement term $\underline{\mathcal{V}}$ can be used for instance. A unique $\underline{d}^{*}$ exists, if $\operatorname{rank}(\underline{A})$ is equal to the number of searched parameters of the transformation vector $\underline{d}$, i.e.

$$
\operatorname{Rank}(\underline{A})=3
$$

and the rank of the composed matrix $\left[\underline{B}^{T} \underline{A}\right]$ is equal to the number of constraints $p$ with $\underline{B} \in \mathbb{R}^{m \times p}[14$, p. 212$]$ i.e.

$$
\operatorname{Rank}\left[\left(\begin{array}{ll}
\underline{B}^{T} & \underline{A}
\end{array}\right)\right]=p .
$$

The covariance matrix of the estimated parameters can be calculated by the inverse of a part of $N$ in (35). If there are $r$ Lagrange multipliers, the rows and columns from $r+1$ to the end of the matrix $\mathrm{N}$ have to be used for covariance calculation. The estimated residuals can be calculated by (31).

Given the estimated ego-pose $\underline{p}_{\text {vehicle }}$ by dead reckoning and the transformation vector $\underline{d}^{*}$ the transition from inaccurate position estimation $\underline{p}_{d r}$ to the corrected 
ego-pose by scan matching $\underline{p}_{s m}$ is calculated using

$$
\underbrace{\left(\begin{array}{l}
x_{s m} \\
y_{s m} \\
\theta_{s m}
\end{array}\right)}_{\underline{p}_{s m}}=\left(\begin{array}{ccc}
\cos (\phi) & -\sin (\phi) & 0 \\
\sin (\phi) & \cos (\phi) & 0 \\
0 & 0 & 1
\end{array}\right) \cdot \underbrace{\left(\begin{array}{l}
x_{d r} \\
y_{d r} \\
\theta_{d r}
\end{array}\right)}_{\underline{p}_{d r}}+\underbrace{\left(\begin{array}{c}
t_{x} \\
t_{y} \\
\phi
\end{array}\right)}_{\underline{d}} .
$$

\subsection{Weighting}

To make the proposed method more robust against noisy data two novel weighting criteria are used. These methods emphasize the scan areas with more detected landmarks and reduce thereby the effect of sparse data through enlargement of their covariance. The following weighting criteria are applied to make the solution of the GHM more plausible.

- Model-dependent weighting: At sample time $k$ there are $m$ detections $p_{i}, i=1,2, \ldots, m$ correspondent with map lines $\mathcal{L}_{r}, r=1,2 \ldots, n$. For modeldependent weighting the detections are weighted according to their distribution in the map. Detections on the same map line can be defined as

$$
\mathcal{N}_{\mathcal{L}_{r}}:=\left\{p_{i} \mapsto \mathcal{L}_{r}, \quad 1 \leq i \leq m\right\}
$$

Therefore, there are $\left|\mathcal{N}_{\mathcal{L}_{r}}\right|$ detections on line $\mathcal{L}_{r}$. Since the matching problem must be more affected from the map areas with dense detections, the map line with the maximum number of correspondent detections with

$$
\mathcal{M}_{\text {map }}=\max \left(\left|\mathcal{N}_{\mathcal{L}_{r}}\right|_{r=1}^{n}\right),
$$

members is fully weighted. It is used as a scale for weighting the detections corresponding to other map lines. To reduce the effect of map features with few correspondent detections on matching the weight is reduced exponentially using

$$
\mathcal{W}_{p_{i}}^{2}=e^{\left(\eta\left(\frac{\mathcal{M}_{m a p}-\left|\mathcal{N}_{\mathcal{L}_{r}}\right|}{\mathcal{M}_{\text {map }}}\right)\right)} \text { with } \eta>0,
$$

where $\eta$ is a tuning parameter. Larger values lead to a faster increase of the function and accordingly the covariance.

- Model-independent weighting: For this weighting method a radius of specified distance $D_{t h}$ around each detection is sought for adjacent detections, so that each point has $G_{p_{i}}$ points in its neighborhood, where

$$
\mathcal{G}_{p_{i}}:=\left\{p_{i}, p_{j} \mid\left\|p_{i}-p_{j}\right\| \leq D_{t h}\right\}
$$

with $i, j=1,2, \ldots, m$. The point with the maximum number of adjacent points with

$$
\mathcal{M}_{\text {det }}=\max _{\mathcal{G}_{p_{i}} \in \underline{P}}\left(\left|\mathcal{G}_{p_{i}}\right|_{i=1}^{m}\right)
$$


members is fully weighted and is used as the scale for weighting another detections. Analogous to the model-dependent weighting an exponential function is used to weaken the influence of sparse detections using

$$
\mathcal{W}_{p_{i}}^{1}=e^{\left(\gamma\left(\frac{\mathcal{M}_{\text {det }}-\left|\mathcal{G}_{p_{i}}\right|}{\mathcal{M}_{\text {det }}}\right)\right)} \text { with } \gamma>0
$$

where $\gamma$ is used for tuning. This method can be also used for filtering by removing detections with a number of adjacent detections under a threshold. Traditionally the Euclidean distance between model and data points are used for weighting [1]. It involves the risk of including outliers due to their small distances from model features. The weighting and outlier recognition by our proposed method is independent from the model. Both weighting methods can be used separately or in combination using

$$
\mathcal{W}_{p i}=\mathcal{W}_{p_{i}}^{1} \cdot \mathcal{W}_{p_{i}}^{2}
$$

for each detection $p_{i}$. To integrate the calculated weights in the GHM the diagonal matrix of weights

$$
\underline{\mathcal{W}}:=\operatorname{diag}\left(\underline{\mathcal{W}}_{1}, \ldots, \underline{\mathcal{W}}_{m}\right) \in \mathbb{R}^{2 m \times 2 m}
$$

is generated with $\underline{\mathcal{W}}_{i}=\mathcal{W}_{i} \cdot \underline{I}$ with $\underline{I} \in \mathbb{R}^{2 \times 2}$ denoting the identity matrix. The covariance matrix $\underline{\Sigma}$ in (35) must be replaced by

$$
\underline{\tilde{\Sigma}}=\underline{\mathcal{W}} \cdot \underline{\Sigma} .
$$

Fig. 7.b visualizes the weighting method for a radar measurement. The radar detection in the center of region $R_{1}$ is fully weighted according to its large number of adjacent detections in a radius of $D_{t h}=1.5 \mathrm{~m}$. On the contrary the detection in the center of region $R_{2}$ is surrounded just by one detection and is less weighted in accordance with the color bar. The radar detections are not spatially equally distributed and this method increases the effect of sensed features with dense detections.

In the next section experimental results are presented applying our GHM-based matching approach and they are compared with some state-of-the-art methods.

\section{$5 \quad$ Evaluation process and experimental results}

This section is composed of a set of experiments to verify the performance and usability of the proposed method. They are compared with some point cloud based matching methods i.e. vanilla ICP, MbICP, PLICP, Fourier-Mellin transform based (FMTM) and NDT-based matching (NDTM). Some tests are conducted in a parking garage with several identical floors, which is regarded as a GNSSrestricted outdoor area. For comparison with dGPS the upper floor with GNSS availability is used while the method is applicable in all floors. The approach is 


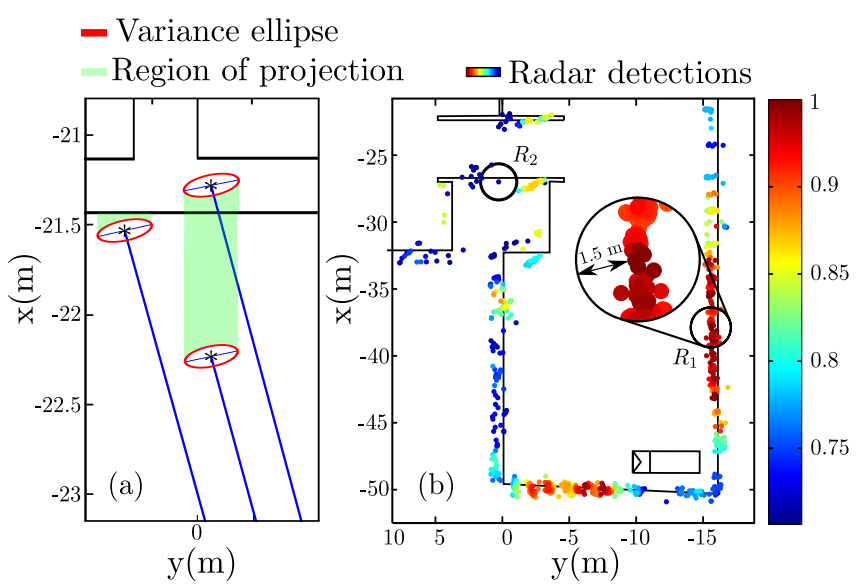

Fig. 7: (a) Three radar detections (black stars), their covariance ellipses and their directions to the vehicle (blue). The green spaces show the possible surface of projection into OSM (black lines). (b) By applying weighting methods the detection in the center of $\mathrm{R} 1$ is more weighted than that in $\mathrm{R} 2$ because of its number of adjacent detections in a certain radius. OSM line on the right side has the most correspondent data and accordingly, they are higher weighted.

also made plausible in a more dynamic environment in urban area. OSMs are provided as the reference map. In Fig. 8 the digitalized blueprint of the parking garage using OSM information is illustrated. OSM contains information about streets, buildings, tracks and much more. However, we extract solely the corner points of perceptible targets for radars (here walls), which are represented as red points. Using these points, the blue lines can be formulated mathematically to be used as feature model for map matching problem. The used test vehicle is equipped with a gyroscope, four wheel-based odometers as standard features and four $77 \mathrm{GHz}$ APTIV short range radars. Each sensor provides every $50 \mathrm{~ms}$ one $2 \mathrm{D}$ measurement in a $150^{\circ}$ field of view with a precision of $1^{\circ}$ in azimuth and $0.1 \mathrm{~m}$ in range up to $40 \mathrm{~m}$ and $0.2 \mathrm{~m}$ in $80 \mathrm{~m}$ distance [13]. For an empirical

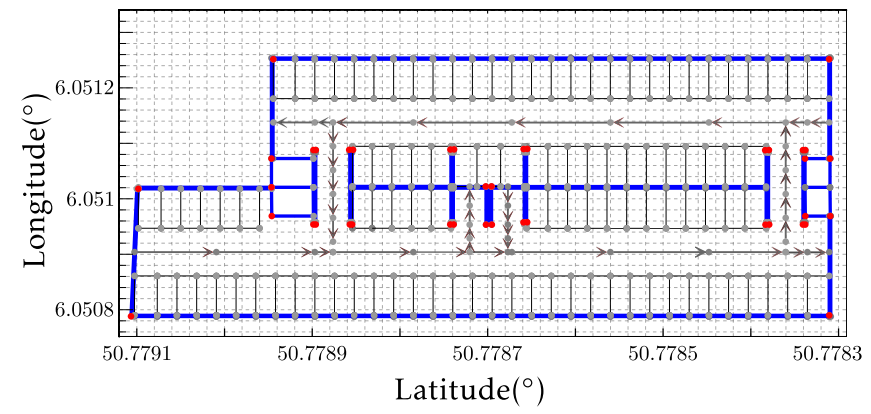

Fig. 8: A parking lot as digitalized blueprint using OpenStreetMap information (gray) and extracted features (red). Blue lines are mathematically defined between each point pair. 
evaluation of proposed method a highly precise differential global positioning system (dGPS) with an accuracy of $\sigma=10 \mathrm{~cm}$ is used. This ground truth is used to compare the results of all ego-pose tracking methods qualitatively and quantitatively using the mean absolute error (MAE) calculated as

$$
M A E=\frac{1}{m} \sum_{i=1}^{m}\left(\left|Q_{d G P S}(i)-Q_{M}(i)\right|\right) .
$$

Here $Q_{d G P S}$ and $Q_{M}$ are the ground truth data and calculated ego-position,

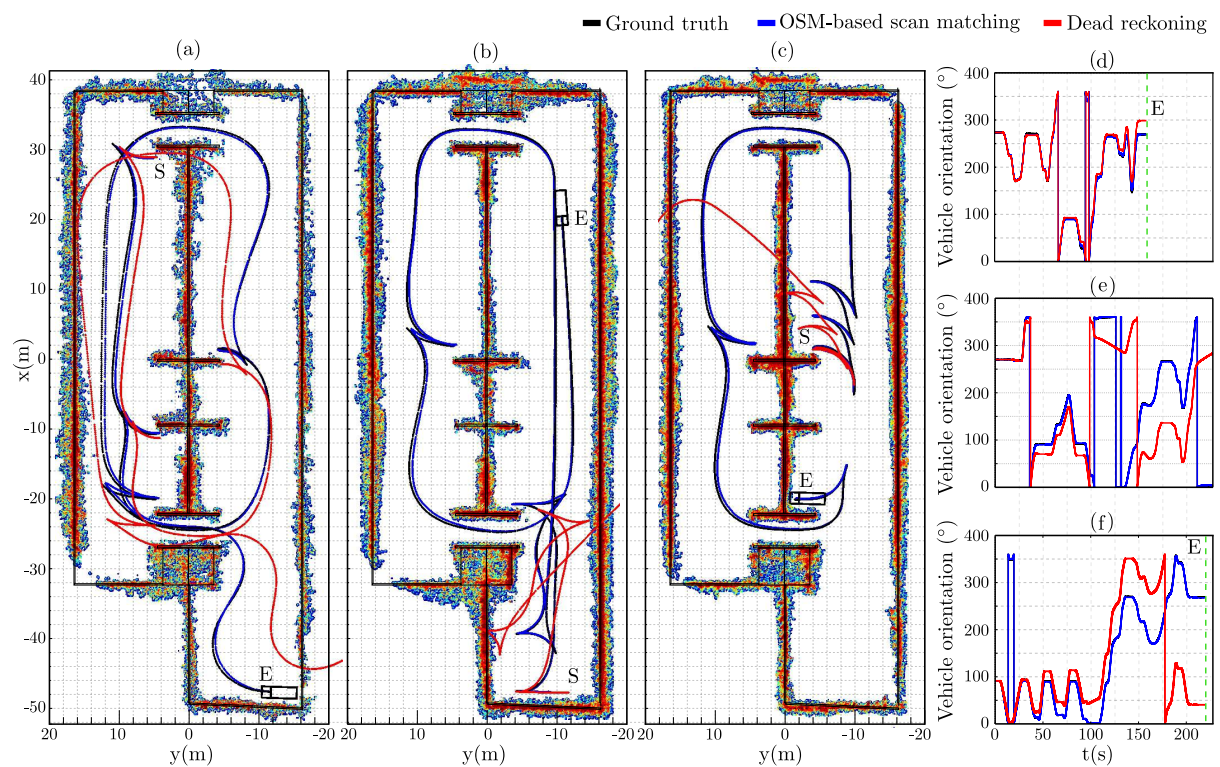

Fig. 9: Evaluating the proposed algorithm by conducting experiments in a parking garage. The letters $\mathrm{S}$ and $\mathrm{E}$ demonstrate the start and end position of drives, respectively. The dGPS trajectory (black), estimated ego-position by dead reckoning (red) and the improved estimation by proposed matching algorithm (blue) are illustrated. The vehicle heading in scenarios (a), (b) and (c) are shown in (d), (e) and (f), respectively. In all scenarios an occupancy grid map is generated based on improved ego-pose by matching, which overlaps the environment representation (black lines).

respectively, and $m$ is the number of measurements. Fig. 9 illustrates the results of three experiments in the parking garage. Driven tracks are curvy to determine how the radar scan matching copes with high dynamic driving maneuvers. The vehicle starts and ends at the positions denoted by S and E, respectively. Fig. 9.a, 9.b and 9.c show the vehicle position trajectory and the Fig. 9.d, 9.e and 9.f the vehicle orientation in these scenarios, respectively. The ground truth data is shown in black, estimated ego-pose by dead reckoning in red and its 
improvement applying proposed matching method in blue. In order to maintain the clarity, the ego-trajectory retained from state-of-the-art methods are not illustrated here and we analyze them later. The more curvatures the driving path contains, the faster fails the dead reckoning to track the vehicle's pose due to the sensor error accumulation over time. All figures depict graphically that the proposed matching method produces accurate displacement estimates, as the blue trajectory is noticeably close to ground truth. Using radar scans an OGM is generated for all scenarios, its overlap with the environment model confirms the accuracy of the proposed scan matcher as well. The qualitative evaluation of GHM-based scan matching (GHM-SM) performance for scenarios in Fig. 9 is represented in Table 1 . It contains the results of the quality levels using (48) and error variances after 2400 measurements. The results are compared with ego-pose estimation purely from dead reckoning (DR) and its improvement with state-ofthe-art matching approaches. All matching methods prevent the divergence of the estimated ego-pose from the ground truth. Using FMTM results in a more inaccurate ego-pose estimations and considerably high error variances resulting in a noisy ego-trajectory. Applying the proposed matching method, the estimation is improved in most cases, which is reflected in the reduction of calculated quality level as well as in error variance. By using the proposed weighting methods (WGHM-SM) the estimation is further improved with a slightly reduced error variance or without its modification. While directly targeting the improvement of ego-localization quality, the robustness as well as the convergence rates of the algorithms have to be analyzed as well. For verifying the convergence rate we consider iterative algorithms i.e. ICP-based methods, which require the correspondences between the map and observations. The convergence is monitored by changing the sum of squares

$$
\mathrm{SOS}=\frac{1}{m} \sum_{i=1}^{m}\left|M_{i}-D_{i}\right|_{2}^{2}
$$

where $D$ and $M$ are the observation point cloud and its correspondent map points, respectively, and $m$ is the number of measurements. Fig. 10.a illustrates the convergence with an initial position error of $6 \mathrm{~m}$ and Fig. 10.b with a initial orientation error of $6^{\circ}$.

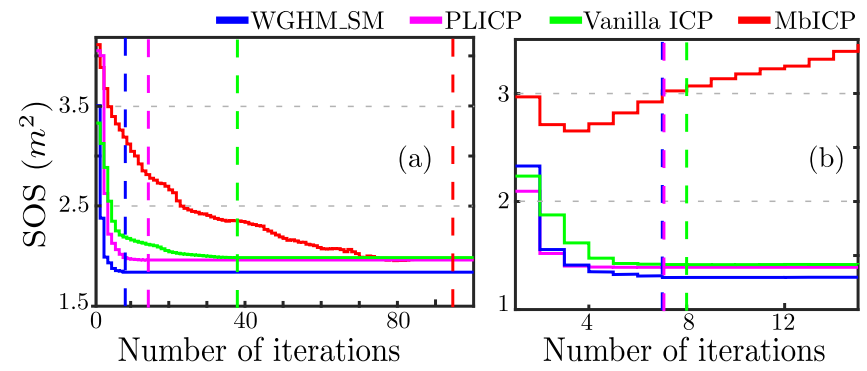

Fig. 10: Convergence rate of scan matching methods for (a) $6 \mathrm{~m}$ initial position error and (b) $6^{\circ}$ initial heading error. 
Table 1: Quality level (QL) calculated using MAE and error variance (var) for position (P) and heading $(\mathrm{H})$ estimation by dead reckoning (DR) and its combination with state-of-the-art methods as well as with proposed technique without using weighting methods (GHM-SM) and after applying them (WGHM-SM) in scenarios a, b and c in Fig. 9. The units for position and heading are meter and degree, respectively.

\begin{tabular}{c|c|c|c|c|c|c|c|c|c|}
\cline { 2 - 9 } \multicolumn{1}{c|}{} & \multicolumn{2}{c|}{ DR } & \multicolumn{2}{c|}{ Vanilla ICP } & \multicolumn{2}{c|}{ MbICP } & \multicolumn{2}{c|}{ PLICP } \\
\cline { 2 - 10 } \multicolumn{1}{c|}{} & QL & var & QL & var & QL & var & QL & var \\
\hline \multirow{3}{*}{$\mathrm{P}$} & $\mathrm{a}$ & 2.16 & 3.40 & 0.325 & 0.024 & 0.29 & 0.031 & 0.32 & 0.022 \\
\hline & $\mathrm{b}$ & 3.5 & 11 & 0.288 & 0.044 & 0.58 & 0.32 & 0.31 & 0.05 \\
\hline $\mathrm{c}$ & 4.65 & 30 & 0.36 & 0.027 & 0.34 & 0.021 & 0.36 & 0.027 \\
\hline $\mathrm{H}$ & $\mathrm{a}$ & 3.20 & 14.9 & 1.41 & 1.067 & 1.458 & 1.1 & 1.43 & 1.07 \\
\hline & $\mathrm{b}$ & 18.5 & 180 & 1.36 & 0.65 & 1.11 & 0.93 & 1.44 & 0.62 \\
\hline & $\mathrm{c}$ & 21.7 & 210 & 1.32 & 0.94 & 1.35 & 1.19 & 1.32 & 0.94 \\
\hline
\end{tabular}

\begin{tabular}{|c|c|c|c|c|c|c|c|c|}
\hline & & & & & & & & \\
\hline & $\mathrm{QL}$ & var & $\mathrm{QL}$ & var & $\mathrm{QL}$ & $\overrightarrow{\mathrm{ar}}$ & $\mathrm{QL}$ & \\
\hline & 0.3 & 0.047 & 0.56 & 0.212 & 30 & 0.0 & 5.21 & \\
\hline & \begin{tabular}{l|l} 
b 0.49
\end{tabular} & 0.15 & .59 & .28 & 23 & 31 & 1 & .0 \\
\hline & \begin{tabular}{l|l} 
c & 0.2
\end{tabular} & & & & & & & \\
\hline & $\begin{array}{lll}\text { a } & 1 .\end{array}$ & & 3.74 & & & & & \\
\hline & b 1 & & 2.79 & & & & 1.04 & \\
\hline & (a) & \begin{tabular}{|l|}
1.11 \\
\end{tabular} & 8 & 4.19 & & 0.7 & 1.0 & 0.7 \\
\hline
\end{tabular}

The first row of the Fig. 11 illustrates the position estimation error and the second row the orientation estimation error versus each position and orientation error. Obviously, PLICP and WGHM-SM are robust enough against an initial pose error in this range. However, WGHM-SM results in a smaller position and orientation error even in the case of large initial pose errors. PLICP has a more precise and quick performance compared with Vanilla ICP and MbICP. However, WGHM-SM converges more quickly after only 8 iterations in Fig. 10.a and has the same convergence rate as PLICP for initial heading noise. It results in both cases in a smaller SOS as PLICP. ICP and MbICP need more time to converge and MbICP fails in case of large initial heading error. From now on, only matchers with best performance are compared, i.e. WGHM-SM, Vanilla ICP and PLICP. In order to verify the robustness of the matchers we run 1681 iteration for each algorithm and add a noise in a certain range to the initial pose. A critical aspect of point cloud based matching algorithms is the run time. Since PLICP converges quicker than Vanilla ICP and has a better performance we compared its run time with run time of WGHM-SM in MATLAB per scan. With $0.02 \mathrm{~s}$ the proposed method is approximately as twice as fast compared to PLICP with a run time of $0.041 \mathrm{~s}$. WGHM-SM is real-time capable and its run frequency is higher than the measuring frequency $(20 \mathrm{~Hz})$. Note that the 
digitalized map has to be processed one step more for ICP-based methods, since the feature map has to be discretized and transformed in a point map. Depending on discretization resolution it can result in a big data volume if the map belongs to a large area e.g. a part of a city. The higher the number of points, the longer ICP-based methods need to find the correspondences. The OSM of the parking garage contains only 50 lines and its transformation in point cloud with a resolution of $0.1 \mathrm{~m}$ results in a point map with 4575 points for this small area.

During these experiments the environment remained static. However, the described algorithm showed to be robust even when the assumption of a static environment is violated like scenarios with people walking, driving and parked
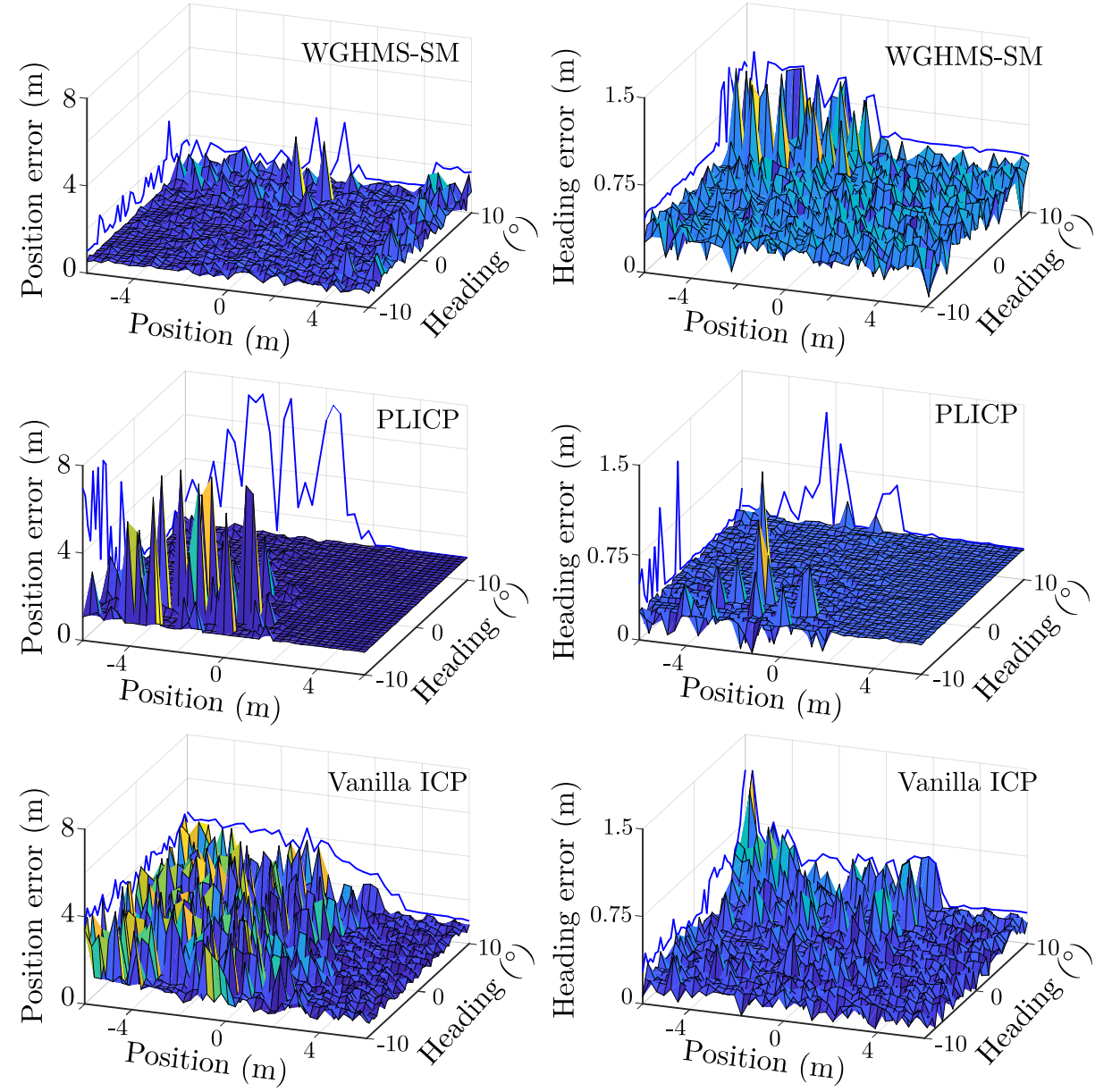

Fig. 11: Robustness against inaccurate initial pose. The first column illustrates position and the second column the heading estimation error for initial position error up to $6 \mathrm{~m}$ and initial heading error up to $10^{\circ}$ on 1681 iterations. Top: WGHM-SM, middle: PLICP, bottom: Vanilla ICP. 


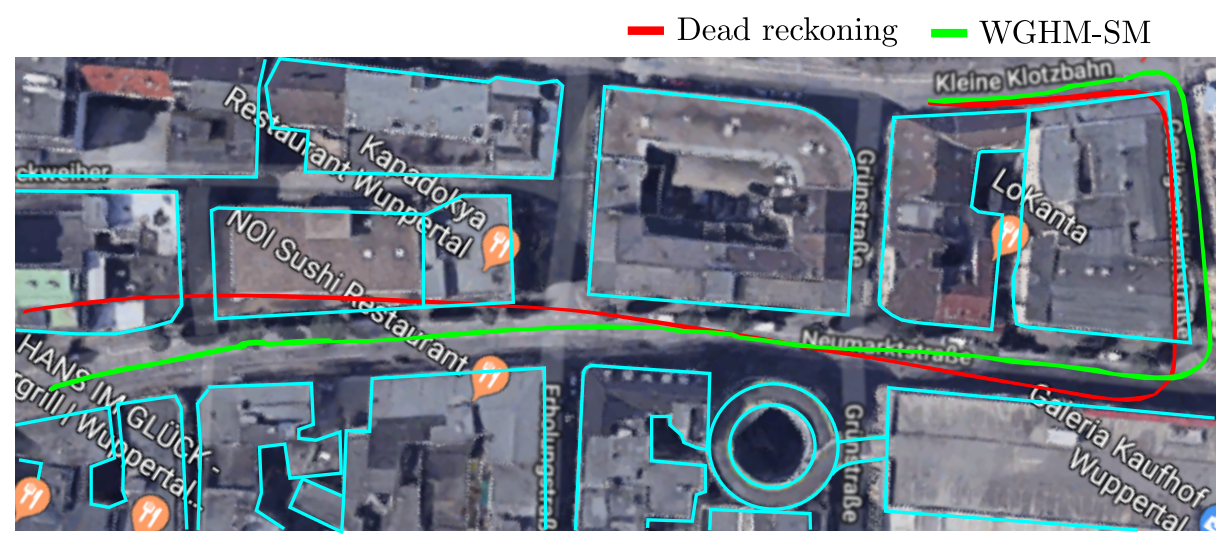

Fig. 12: Ego-position estimation without using WGHM-SM (red) and after applying it (green) in a real scenario in urban area. Blue lines show the edges of the buildings. Photo: Imagery (c)2018 Google, Map data (c)2018 GeoBasis-DE/BKG (c)2009).

vehicles in urban environments. Fig. 12 shows an experiment in urban environment. The ego-position trajectory estimated by DR has an overlap with the buildings (red) which is not the case using matching algorithm (green). Fig. 13 illustrates another evaluation of the proposed map matching in the area surrounding the APTIV building in Wuppertal. The OSM lines are shown in blue which contain information of buildings, fences and walls. During this experiment the environment is dynamic. There are standing and walking pedestrians (white stars) as well as parked and driving vehicles which are shown as yellow and red stars, respectively. There are some stationary objects such as containers covering the buildings which are not registered in the map. The red trajectory shows the result of self-localization without applying map matching and the green trajectory after its application. As in the experiment in Fig. 12 there is an overlap between estimated ego-trajectory and the map lines by using DR (red). WGHM-SM is evaluated with 182416 measurements in different scenarios. The quantitative results confirm the performance of the proposed scan matcher in static as well as in dynamic environments. It is robust against inaccurate initial pose, converges faster that ICP-based methods and causes in most cases a smaller estimation error.

\section{Conclusion and future work}

In this paper a fast scan matching algorithm is proposed to improve the inaccurate ego-pose estimated basically by dead reckoning using a map which consists of mathematically described lines. The vehicle displacement caused by drift and bias of mechanical motion sensors is minimized by the matching of radar measurements to features of the environment representation. Therefore, this matching method belongs to the class of point-to-feature matching algorithms. Scan 


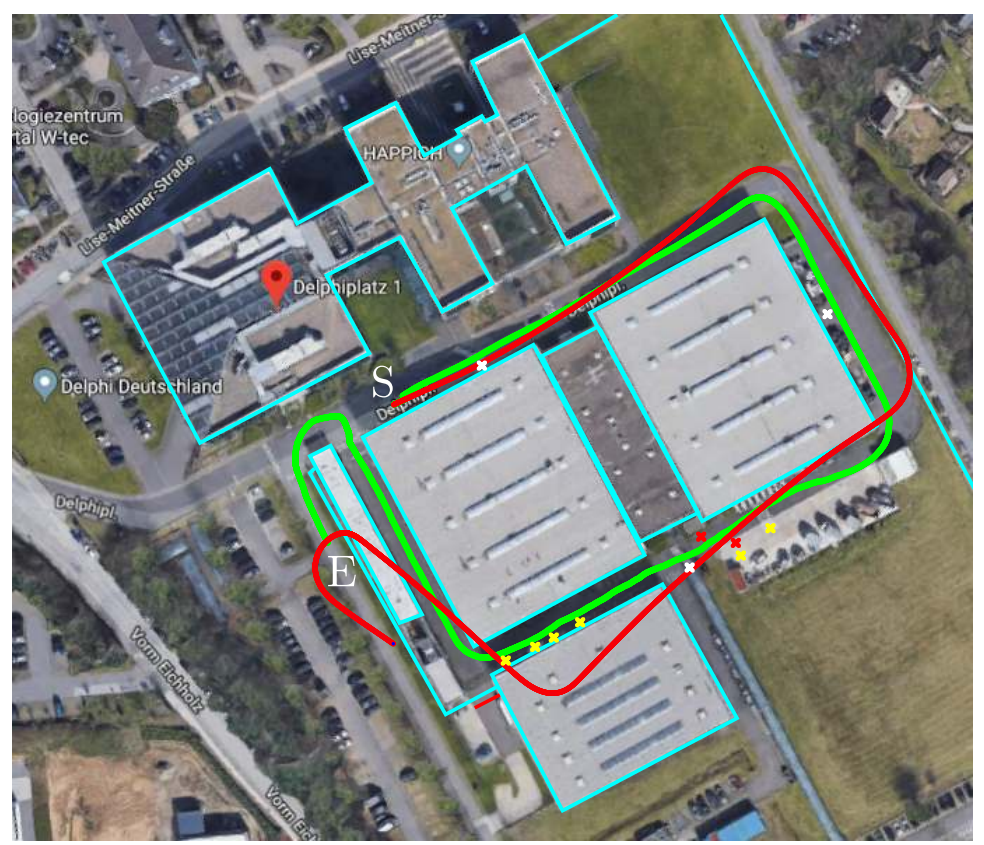

Fig. 13: Validation of the proposed map matching in a outdoor dynamic environment with pedestrians, parked and driving vehicles. Their rough places are shown as white, yellow and red stars, respectively. Blue lines show the edges of the buildings. The red and green trajectories are generated without and with applying the proposed map matching approach, respectively. The letters $\mathrm{S}$ and E show the start and end point. Photo: Imagery (C)2018 Google, Map data (C)2018 GeoBasis-DE/BKG (C)2009).

matching using radar measurements is challenging because of the sensor noise, measurement sparseness and multiple path propagations. The presented method deals with this problem using appropriate filtering, novel weighting methods and considering radar measurement uncertainties in an iterative optimization problem by using the Gauß-Helmert-Model. Various experiments have been carried out in outdoor areas with different driving dynamic maneuvers to evaluate the matching method against dead reckoning and other matching methods in different scenarios. Experimental results confirm qualitatively and quantitatively the accurate and robust performance of scan matching for improving the selflocalization precision.

Future work is expected to estimate the vehicle initial pose by applying probabilistic methods for global localization and matching of 3D radar measurements. Furthermore, a classification of the stationary radar observations to achieve a better data association could help to improve the algorithm performance. For instance the stationary observations caused by parked vehicles in urban environment can be classified to avoid their association with the environment model. Using environment models with more object information such as highly accurate high-definition (HD) maps is another possibility. These maps are generated 
using Lidar, cameras, dGPS, etc. and they do not only contain information of walls and buildings but of many other objects such as trees, guard rails, etc. In this case the map matching algorithm has to be extended to cope not only with a model consisting of lines.

\section{References}

1. P. Bergström and O. Edlund. Robust registration of point sets using iteratively reweighted least squares. Comput Optim Appl. Springer Science+Business Media New York, Feb. 2014.

2. P. Besl and N. McKay. A method for registration of 3-d shapes. IEEE Trans. on Pattern Analysis and Machine Intelligence, (2):239-256, Feb. 1992.

3. Peter Biber and Wolfgang Straßer. The normal distributions transform: A new approach to laser scan matching. Proceedings IEEE/RSJ International Conference on Intelligent Robots and Systems, Oct. 2003.

4. J. Borenstein and L. Feng. Correction of systematic odometry errors in mobile robots. International Conference on Intelligent Robots and Systems, Aug. 1995.

5. Michael Bosse and Jonathan Roberts. Histogram matching and global initialization for laser-only slam in large unstructured environments. IEEE International Conference on Robotics and Automation, Apr. 2007.

6. A. Censi. An ICP variant using a point-to-line metric. IEEE International Conference on Robotics and Automation, May 2008.

7. Andrea Censi, Luca Iocchi, and Giorgio Grisetti. Scan matching in the hough domain. IEEE ICRA, 2005.

8. Paul Checchin, Franck Gérossier, Christophe Blanc, Roland Chapuis, and Laurent Trassoudaine. Radar scan matching slam using the fourier-mellin transform. Howard A., Iagnemma K., Kelly A. (eds) Field and Service Robotics. Springer Tracts in Advanced Robotics, 62:151-161, 2010.

9. M. Cole and P. M. Newman. Using laser range data for 3d slam in outdoor environments. IEEE International Conference on Robotics and Automation (ICRA), pages 1556-1563, May 2006.

10. Martin A. Fischler and Robert C. Bolles. Random sample consensus: A paradigm for model fitting with applications to image analysis and automated cartography. Commun. ACM, 24(6):381-395, June 1981.

11. Gene H. Golub and Charles F. van Loan. An analysis of the total least squares problem. SIAM Journal on Numerical Analysis, 17(6):883-893, 1980.

12. M. Haklay and P. Weber. Openstreetmap: User-generated street maps. Pervasive Computing, IEEE CS, (4), 2008.

13. Alexander Ioffe. Situation awareness by radar technology. SAE 2016 ADAS to Automated Driving Symposium, Nov. 2016.

14. K.R. Koch. Parameterschätzung und Hypothesentests in linearen Modellen: Dümmlers Typoscripts. Dümmler, 1980.

15. H. Lategahn and C. Stiller. Vision-only localization. IEEE Transactions on Intelligent Transportation Systems, (3):1246-1257, Feb. 2014.

16. L. Lenzmann and E. Lenzmann. Strenge Auswertung des nichtlinearen GaußHelmert-Modells. pages 68-73, Jan. 2003.

17. Jesse Levinson and Sebastian Thrun. Robust vehicle localization in urban environments using probabilistic maps. IEEE International Conference on Robotics and Automation, 2010. 
18. Feng Lu and E. Milios. Robot pose estimation in unknown environments by matching $2 \mathrm{~d}$ range scans. IEEE Conference on Computer Vision and Pattern Recognition, Jun. 1994.

19. J. Minguez, L. Montesano, and F. Lamiraux. Metric-based iterative closest point scan matching for sensor displacement estimation. IEEE Transactions on Robotics, (5):1047-1054, Oct. 2006.

20. David Nistér, Oleg Naroditsky, and James Bergen. Vision odometry. IEEE Computer Society Conference on Computer Vision and Pattern Recognition, Jul. 2004.

21. J. Nocedal and S. Wright. Numerical Optimization. relax Springer Science+Business Media, LLC, 2006.

22. Edwin B. Olson. Real-time correlative scan matching. IEEE International Conference on Robotics and Automation, May 2009.

23. Ahmad Pishehvari, Uri Iurgel, Stephanie Lessmann, Lutz Roese-Koerner, and Bernd Tibken. Radar scan matching using navigation maps. pages 204-211, 02 2019.

24. Lutz Roese-Koerner. Convex Optimization for Inequality Constrained Adjustment Problems. PhD thesis, Deutsch Geodätische Kommission der Bayerischen Akademie der Wissenschaten, München, Germany, 2015.

25. Philipp Ruchti, Bastian Steder, Michael Ruhnke, and Wolfram Burgard. Localization on openstreetmap data using a 3d laser scanner. IEEE International Conference on Robotics and Automation, may 2015.

26. Sebastian Thrun, Wolfram Burgard, and Dieter Fox. Probabilistic Robotics (Intelligent Robotics and Autonomous Agents). The MIT Press, 2005.

27. M. Tomono. A scan matching method using euclidean invariant signature for global localization and map building. IEEE international Conference on Robotics and Automation, lA, pp. 886-871, Apr. 2004.

28. P.H.S. Torr and A. Zisserman. Mlesac. Comput. Vis. Image Underst., 78(1):138156, April 2000. 